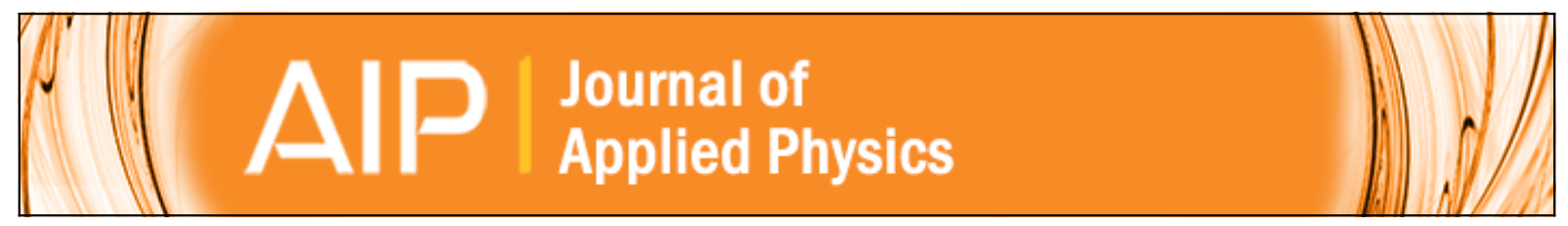

Infrared organic light emitting diodes using neodymium tris-(8-hydroxyquinoline)

O. M. Khreis, R. J. Curry, M. Somerton, and W. P. Gillin

Citation: Journal of Applied Physics 88, 777 (2000); doi: 10.1063/1.373803

View online: http://dx.doi.org/10.1063/1.373803

View Table of Contents: http://scitation.aip.org/content/aip/journal/jap/88/2?ver=pdfcov

Published by the AIP Publishing

Articles you may be interested in

White light emission from exciplex using tris-(8-hydroxyquinoline)aluminum as chromaticity-tuning layer

Appl. Phys. Lett. 78, 3947 (2001); 10.1063/1.1379788

Radiative recombination mechanisms in aluminum tris(8-hydroxyquinoline): Evidence for triplet exciton recombination

J. Appl. Phys. 88, 781 (2000); 10.1063/1.373802

Electroabsorption spectroscopy on tris-(8-hydroxyquinoline) aluminum-based light emitting diodes

J. Appl. Phys. 86, 4978 (1999); $10.1063 / 1.371469$

$1.54 \mathrm{~m}$ electroluminescence from erbium (III) tris(8-hydroxyquinoline) (ErQ)-based organic light-emitting diodes

Appl. Phys. Lett. 75, 1380 (1999); 10.1063/1.124700

Humidity-induced crystallization of tris (8-hydroxyquinoline) aluminum layers in organic light-emitting devices Appl. Phys. Lett. 72, 756 (1998); 10.1063/1.120867

\section{High-Voltage Amplifiers}

- Voltage Range from $\pm 50 \mathrm{~V}$ to $\pm 60 \mathrm{kV}$

- Current to 25A

\section{Electrostatic Voltmeters}

- Contacting \& Non-contacting

- Sensitive to $1 \mathrm{mV}$

- Measure to $20 \mathrm{kV}$
ENABLING RESEARCH AND

INNOVATION IN DIELECTRICS,

ELECTROSTATICS, MATERIALS, PLASMAS AND PIEZOS

TRek www.trekinc.com 


\title{
Infrared organic light emitting diodes using neodymium tris-(8-hydroxyquinoline)
}

\author{
O. M. Khreis \\ Hijjawi Faculty for Engineering and Technologies, Yarmouk University, Irbid, Jordan \\ R. J. Curry, M. Somerton, and W. P. Gillin \\ Department of Physics, Queen Mary and Westfield College, University of London, London E1 4NS, \\ United Kingdom
}

(Received 6 March 2000; accepted for publication 5 April 2000)

\begin{abstract}
We have studied the photoluminescence and electroluminescence of neodymium tris-(8-hydroxyquinoline) and have found evidence, from the Stark splitting of the neodymium emission, for two isomers of the molecule. Following sublimation it appears that one of these isomers predominates. Photoluminescence can be excited through absorption into the organic ligands and there appears to be efficient coupling between the singlet and triplet exciton levels in the ligand and the internal levels of the neodymium. We can obtain bright infrared electroluminescence from the intraatomic levels within the neodymium at wavelengths of 900, 1064, and $1337 \mathrm{~nm}$. (C) 2000 American Institute of Physics. [S0021-8979(00)00714-3]
\end{abstract}

\section{INTRODUCTION}

Near infrared luminescence from organic materials has been attracting interest recently due to the potential of producing electroluminescent devices on a range of substrates. Recent work has shown that electroluminescence can be obtained, at various wavelengths, from rare-earth containing organometallic compounds. For example, europium and terbium ${ }^{1,2}$ have been used to obtain sharp visible luminescence and neodymium and erbium to produce infrared luminescence. ${ }^{3,4}$ The potential for the infrared emitters include integrating the materials directly onto silicon to produce devices for interchip communications or in the case of erbium containing molecules for integrated $1.5 \mu \mathrm{m}$ sources for telecommunications applications. ${ }^{4}$

In recent work we have shown that not only can we obtain $1.5 \mu \mathrm{m}$ photoluminescence from erbium tris(8hydroxyquinoline) (ErQ), ${ }^{4}$ but we can also produce electroluminescent diodes grown on indium-tin-oxide (ITO) substrates using ErQ as the electron transporting and emitting layer and $\mathrm{N}, \mathrm{N}^{\prime}$-diphenyl-N, $\mathrm{N}^{\prime}$-bis(3-methylphenyl)-1, $1^{\prime}$-biphenyl-3,3'-diamine (TPD) as the hole transporting layer. ${ }^{5}$ In this article we describe detailed photoluminescence and electroluminescence experiments on neodymium tris-(8hydroxyquinoline).

\section{EXPERIMENTAL METHOD}

Neodymium tris(8-hydroxyquinoline) was synthesized by dissolving neodymium chloride in de-ionized water and 8-hydroxyquinoline in an $80 \%$ methanol $20 \%$ de-ionized water solution. The first solution was slowly added to the second over a period of $2 \mathrm{~h}$ while undergoing stirring at ambient temperature. Further methanol was added and the solution left stirring overnight. The solution was then filtered and the remaining precipitate washed with methanol, at $-25^{\circ} \mathrm{C}$, to try to remove any remaining 8 -hydroxyquinoline. The resulting substance was then dissolved in methanol at ambient temperature and hexane was added until a fine precipitate was seen. The solution was then placed in a freezer to cool before the precipitate was finally collected. Liquid chromatography was then used to ascertain that only one material was present in the precipitate.

For the photoluminescence measurements either a 400 $\mathrm{nm}$ thick sublimed film or a quantity of the bulk powder in a quartz curvette was placed inside a continuous flow cryostat, which allowed the temperature to be varied between 4 and $300 \mathrm{~K}$. Photoluminescence was excited using various lines from a UV enhanced argon ion laser, dispersed in a $1 \mathrm{~m}$ spectrometer and detected using an S-20 photomultiplier for the visible wavelengths or a liquid nitrogen cooled Ge detector for the infrared. All spectra were corrected for the response of the system. To obtain the absorption spectra $0.1 \mathrm{~g}$ of neodymium chloride was dissolved in $50 \mathrm{ml}$ of methanol. The absorption spectra were obtained using an Hitachi U-3000 spectrophotometer.

All sublimed films and diodes were fabricated at a base pressure of $\sim 10^{-6}$ Torr. Diodes were formed on to cleaned ITO coated glass substrates and consisted of a $50 \mathrm{~nm}$ layer of TPD as a hole transporting layer followed by $60 \mathrm{~nm}$ of the NdQ, all deposited at 2-3 $\AA /$ s. The top contact was a $200 \mathrm{~nm}$ layer of aluminum.

\section{RESULTS AND DISCUSSION}

A sample of the bulk powder was used to measure the visible, ligand related, photoluminescence using both 351 and $457 \mathrm{~nm}$ excitation wavelengths as a function of temperature. Figure 1 shows the $80 \mathrm{~K}$ photoluminescence for these two excitation lines along with the absorption curve for a solution of neodymium chloride in methanol. As the temperature is increased the shape of the photoluminescence does not significantly change, although the intensity of the photoluminescence reduces slightly; by approximately $10 \%$ for the $457 \mathrm{~nm}$ excitation and $\sim 30 \%$ for the $351 \mathrm{~nm}$ excita- 


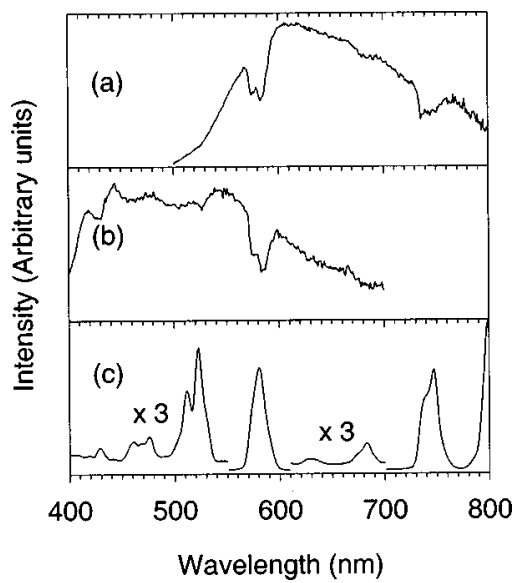

FIG. 1. The $80 \mathrm{~K}$ visible photoluminescence from bulk NdQ powder excited using the (a) $457 \mathrm{~nm}$ line and the (b) $351 \mathrm{~nm}$ line from an argon ion laser. (c) shows the absorption spectra for $\mathrm{Nd}^{3+}$ ions obtained from a solution of $\mathrm{NdCl}_{3}$ in methanol.

tion. From the absorption spectra of NdQ, shown as an inset to Fig. 2, both of these excitation wavelengths are within the ligand absorption bands so it is reasonable to believe that the visible photoluminescence is originating from the ligands. The detailed fine peak structure visible in these spectra are, however, not characteristic of organic materials. By comparing the positions of the troughs in the visible photoluminescence with the peaks in the absorption of a $\mathrm{NdCl}_{3}$ solution [Fig. 1(c)] we can ascribe these sharp features to absorption of the photoluminescence by the $\mathrm{Nd}^{3+}$ ions within the molecules. The presence of these absorption lines in the photoluminescence spectra again support our assumption that it is the ligand which is responsible for the broad visible photoluminescence.

It can be seen from Fig. 1 that there are significant differences between the visible photoluminescence excited using the two wavelengths. Although absolute peak positions for the emission cannot be determined, due to the presence of the absorption lines, it appears that the photoluminescence peak for the $351 \mathrm{~nm}$ excited sample is $\sim 450-550 \mathrm{~nm}$, whereas for the $457 \mathrm{~nm}$ excited luminescence the peak is at $\sim 600-650 \mathrm{~nm}$. These results are in qualitative agreement with results we have obtained for AlQ. ${ }^{6}$ In that work we investigated the luminescence of AlQ as a function of excitation wavelength and temperature and noted dramatic shifts

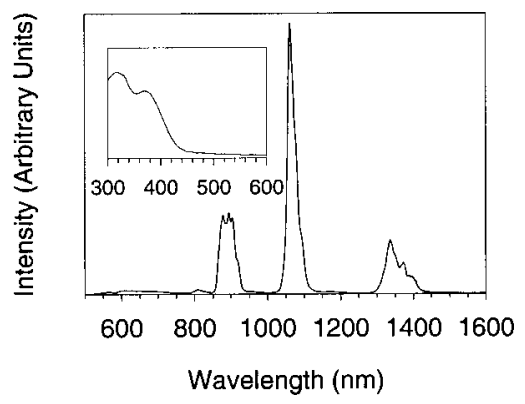

FIG. 2. The $80 \mathrm{~K}$ photoluminescence from bulk NdQ powder excited at 457 $\mathrm{nm}$. The spectra has been corrected for the response of the system. The inset shows the absorption spectra for NdG. in the photoluminescence spectra. These shifts were attributed to longer wavelength excitations being directly absorbed to form triplet excitons, some of which could radiatively recombine giving photoluminescence at $\sim 600 \mathrm{~nm}$. We also noted that the long wavelength tail observed in the UV excited photoluminescence of AlQ but not in the electroluminescence could be attributed to triplet exciton recombination.

If the highest occupied molecular orbital to lowest unoccupied molecular orbital separation is not strongly modified by the central ion we would expect NdQ to give a roughly similar visible luminescence to AlQ. However, even ignoring the effects of the absorption lines, the UV excited NdQ photoluminescence appears to be much broader than that for AlQ. This broadening could be attributed to greater radiative recombination of triplets, which could be caused by the greater spin-orbit interaction caused by the larger mass of $\mathrm{Nd}$ compared to Al. These triplets, having a lower energy than the singlet excitons, would result in emission in the tail of the singlet spectra and hence the observed broadening.

For the $457 \mathrm{~nm}$ excitation the shift in the photoluminescence peak is very much more pronounced than in AlQ. From the photoluminescence there appears to be almost no singlet recombination visible, the $\sim 500 \mathrm{~nm}$ peak, and very little dependence of either peak position or intensity with temperature, $\sim 10 \%$ increase in intensity between 300 and $80 \mathrm{~K}$.

It should be stressed that independent of excitation wavelength the visible photoluminescence from this material is exceptionally weak $\left(\sim 1\right.$ in $\left.10^{3}\right)$ when compared to AlQ measured under identical conditions.

Figure 2 shows the photoluminescence of bulk NdQ recorded from $500 \mathrm{~nm}$ to $1.6 \mu \mathrm{m}$ using $100 \mathrm{~mW}$ of $457 \mathrm{~nm}$ excitation. This figure is a composite of the visible range collected using the photomultiplier and the infrared range collected using the Ge detector. The spectra have been scaled to match where they overlap to demonstrate the relative intensities of the two regions. From Fig. it can be seen that, compared to the weak visible luminescence, there are three strong infrared emission lines centred at $\sim 900,1064$, and $1337 \mathrm{~nm}$ which correspond to the ${ }^{4} F_{3 / 2} \rightarrow{ }^{4} I_{9 / 2},{ }^{4} F_{3 / 2}$ $\rightarrow{ }^{4} I_{11 / 2}$, and ${ }^{4} F_{3 / 2} \rightarrow{ }^{4} I_{13 / 2}$ transitions, respectively. The observation of this very strong luminescence from the intraatomic neodymium levels coupled with very weak luminescence from the organic ligands suggests very efficient energy coupling between the ligands and the ion. This is supported by the observation of $\mathrm{Nd}$ absorption lines in the ligand luminescence, which show that the singlet and triplet levels in the ligands are resonant with levels within the $\mathrm{Nd}^{3+}$ ion.

Figures 3 and 4 show detailed low temperature luminescence spectra for the ${ }^{4} F_{3 / 2} \rightarrow{ }^{4} I_{9 / 2}$ and ${ }^{4} F_{3 / 2} \rightarrow{ }^{4} I_{11 / 2}$ transitions, respectively, recorded from both bulk NdQ powder and from $400 \mathrm{~nm}$ films of sublimed NdQ. Figure 3 shows the $5 \mathrm{~K}$ spectra from the ${ }^{4} F_{3 / 2} \rightarrow{ }^{4} I_{9 / 2}$ transition, for the bulk powder and ten peaks can be clearly resolved. These peaks are due to the Stark splitting of the ${ }^{4} I_{9 / 2}$ level due to the local electric field seen by the Nd atom. NdQ would be expected to form two isomers, fac and mer, which would have $\mathrm{C}_{3}$ and $\mathrm{C}_{1}$ symmetry respectively. These two isomers would leave 


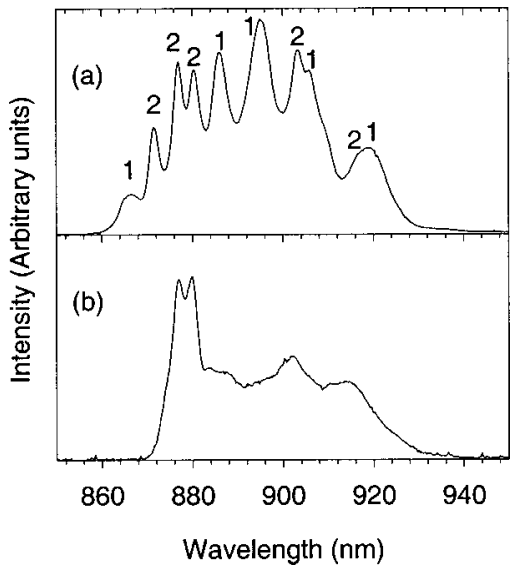

FIG. 3. The $5 \mathrm{~K}$ photoluminescence from the ${ }^{4} F_{3 / 2} \rightarrow{ }^{4} I_{9 / 2}$ transition within the $\mathrm{Nd}^{3+}$ ion for: (a) bulk NdQ and (b) a $400 \mathrm{~nm}$ sublimed film. The peaks marked 1 and 2 in (a) are believed to be due to the different isomers with 2 being the predominant peaks in the sublimed film.

the $\mathrm{Nd}^{3+}$ ion in sites of $\mathrm{C}_{3}$ or $\mathrm{C}_{1}$ symmetry. For the ${ }^{4} I_{9 / 2}$ level both of these site symmetries would result in the Stark splitting producing five levels, so the presence of ten peaks in the bulk NdQ spectrum would suggest that the powder is a mixture of the two isomers. For the sublimed film it is only possible to clearly resolve five peaks, although there is probably some contribution to the background from at least one of the other peaks, which suggests that this layer may consist of mainly one isomer. Although these results appear to show that mainly one isomer is present in the sublimed films, we are not able to detect which of the two isomers it is. It is also not clear what the mechanism resulting in the deposition of a single isomer during evaporation is. It could be that the two isomers have very different sublimation temperatures, causing one to sublime preferentially or it may be that one of the isomers can be transformed with temperature, however, this will need further study. For the ${ }^{4} F_{3 / 2} \rightarrow{ }^{4} I_{11 / 2}$ transition the difference is not as clear (Fig. 4). For this level, due to the Stark splitting, we should see six transitions for each isomer. However, for the bulk NdQ sample we only observe, at most, eight peaks and at least five of these are also present in the sublimed film. While some of the transitions present in the

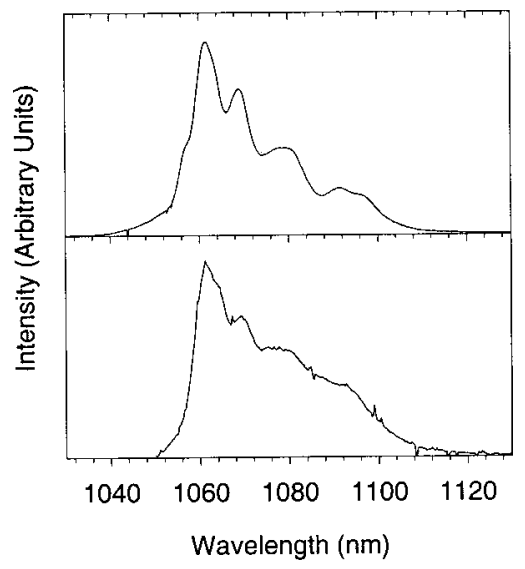

FIG. 4. The $5 \mathrm{~K}$ photoluminescence from the ${ }^{4} F_{3 / 2} \rightarrow{ }^{4} I_{11 / 2}$ transition within the $\mathrm{Nd}^{3+}$ ion for: (a) bulk NdQ and (b) a $400 \mathrm{~nm}$ sublimed film.

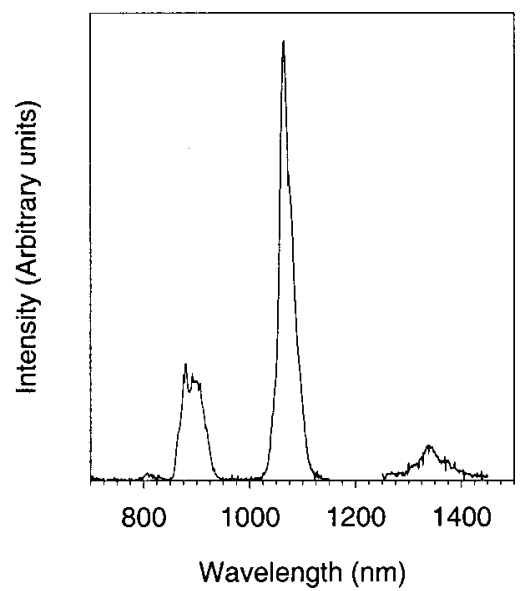

FIG. 5. The $300 \mathrm{~K}$ electroluminescence from a ITO/TPD/NdQ/Al diode.

bulk powder are not present in the sublimed film and are probably due to the second isomer we suggest that some of the detail is hidden due to the high intensity of these levels, which is resulting in them being quite broad. It should also be noted that the positions of the peaks need not necessarily be different for the two isomers and this may be an additional factor in the splitting not being as clear for this level as for the ${ }^{4} F_{3 / 2} \rightarrow{ }^{4} I_{9 / 2}$ transition.

Figure 5 shows the infrared electroluminescence obtained from an ITO/TPD/NdQ/Al diode at an operating voltage of $30 \mathrm{~V}$, which corresponded to a current through the diode of $78 \mathrm{~mA} / \mathrm{cm}^{2}$. This diode showed no visible electroluminescence but strong infrared luminescence centred at $\sim 900,1060$, and $1320 \mathrm{~nm}$ as seen in the photoluminescence. As the electroluminescence spectra were recorded at $300 \mathrm{~K}$, the peaks are therefore somewhat broader than for the spectra shown in Fig. 3 and 4. However, close investigation of the ${ }^{4} F_{3 / 2} \rightarrow{ }^{4} I_{9 / 2}$ transition shows that it looks more like the photoluminescence of the sublimed film as opposed to the bulk powder with a number of the peaks being less clearly resolved. This is not surprising in light of our explanation of the differences in the photoluminescence between the sublimed films and the bulk powder. Figure 6 shows the intensity of the electroluminescence, at $1064 \mathrm{~nm}$, as a function of the current density through the diode. It can be clearly seen that the diodes start emitting at a current density of $\sim 0.23$

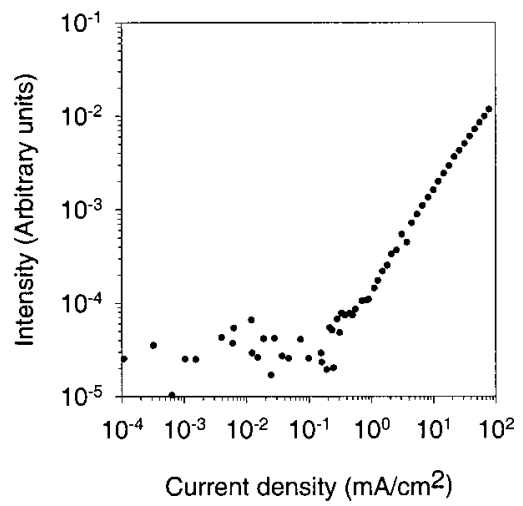

FIG. 6. The electroluminescence intensity at $1064 \mathrm{~nm}$ as a function of the current density through the diode. 
$\mathrm{mA} / \mathrm{cm}^{2}$, which corresponds to a drive voltage of $\sim 13 \mathrm{~V}$. It can also be seen that once the diodes switch on there is a linear increase in intensity with current through the diodes. The current-voltage $(I-V)$ characteristics of these diodes showed a turn on voltage in forward bias of $\sim 13 \mathrm{~V}$ and no breakdown in reverse bias up to voltages of $-30 \mathrm{~V}$. While the $13 \mathrm{~V}$ turn on voltage is quite high, we have made no attempt to optimize these diodes, through oxygen plasma treatment of the ITO or using low work function metals and we see no fundamental reasons why this could not be reduced in the future.

\section{CONCLUSIONS}

In conclusion we have studied the photoluminescence of neodymium tris-(8-hydroxyquinoline) and have found evidence, from the Stark splitting of the neodymium emission, for two isomers of the molecule. Following sublimation it appears that one of these isomers predominates. Photoluminescence can be excited through absorption into the organic ligands and there appears to be efficient coupling between the singlet and triplet exciton levels in the ligand and the internal levels of the neodymium. We can obtain bright infrared electroluminescence from the intra-atomic levels within the neodymium.

\footnotetext{
${ }^{1}$ J. Kido, H. Hayase, K. Hongawa, K. Nagai, and K. Okuyama, Appl. Phys. Lett. 65, 2124 (1994).

${ }^{2}$ J. Kido, W. Ikeda, M. Kimura, and K. Nagai, Jpn. J. Appl. Phys., Part 2 35, L394 (1996).

${ }^{3}$ Y. Kawamura, Y. Wada, Y. Hasegawa, M. Iwamuro, T. Kitamura, and S. Yanagida, Appl. Phys. Lett. 74, 3245 (1999).

${ }^{4}$ W. P. Gillin and R. J. Curry, Appl. Phys. Lett. 74, 798 (1999).

${ }^{5}$ R. J. Curry and W. P. Gillin, Appl. Phys. Lett. 75, 1380 (1999).

${ }^{6}$ R. J. Curry and W. P. Gillin, J. Appl. Phys. 88, 781 (2000).
} 